\title{
Diaphragmatic fatigue and blood flow distribution in shock charis Rousos mD
}

\section{Blood flow}

The classic notion that the respiratory muscles do not receive marked blood flow and do not consume large amounts of oxygen has been challenged recently both theoretically and experimentally.,2 During normotensive states the diaphragm may receive up to $200-400 \mathrm{ml} \cdot 100 \mathrm{~g}^{-1} \cdot \mathrm{min}^{-1}$ blood during inspiratory resistive load or electrophrenic stimulation. ${ }^{3.4}$ During shock, while the blood flow to all other parts of the body is reduced, the diaphragm still receives $50 \mathrm{ml} \cdot 100 \mathrm{~g}^{-1} \cdot \mathrm{min}^{-1}$ when the blood pressure is around $50 \mathrm{mmHg}$ and the cardiac output is about $30-40$ per cent of control.

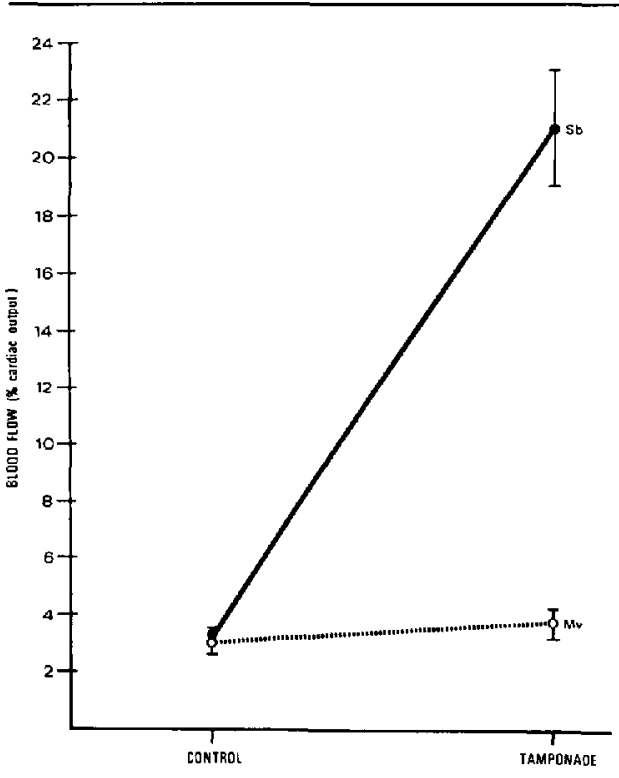

FIGURE I Total respiratory muscle blood flow during control and tamponade in the two groups of dogs expressed as a percentage of cardiac output. Fractional distribution of cardiac output to the respiratory muscles increased more than fivefold during tamponade in the $\mathrm{Sb}$ group.
The latter was a finding in animal experiments in which we have shown that the respiratory muscles of spontaneously breathing animals with a low cardiac output produced by pericardial tamponade or septic shock receive up to 20 per cent of the cardiac output as compared with three per cent when the animals were paralyzed and ventilated ${ }^{5,6}$ (Figure 1)

The large share of the cardiac output taken up by the respiratory muscles in spontaneously breathing animals resulted in reduced blood flow to the brain, the liver and other skeletal muscles as compared with mechanically ventilated animals with a similar reduction in cardiac output ${ }^{5,6}$ (Figure 2).

Of particular clinical importance also are recent findings that the respiratory muscles receive a large amount of cardiac output in an animal model in which septic shock was produced after injection of $E$. coli endotoxin. ${ }^{6}$ In this experiment we observed that, despite a reduction in cardiac output and decrease in blood pressure, the respiratory muscles, and particularly the diaphragm, received an inordinate amount of blood, depriving the rest of the body of adequate energy supply. Again, mechanical ventilation frees blood which is redistributed to other vital organs (Figure 3).

It is predictable that in states of low cardiac output, if the respiratory muscles receive an inordinately large amount of blood and deprive the rest of the body, anerobic metabolism will take place, lactate will be produced, and metabolic acidosis will develop. This prediction has been also confirmed in experiments of cardiogenic (Figure 4) and septic shock; 5,6 however, the important observation in these experiments was that lactate was produced,

Department of Medicine, McGill University and Critical Care Section, Royal Victoria Hospital, 687 Pine Avenue West, Montréal, Quebec H3A 1A1. 


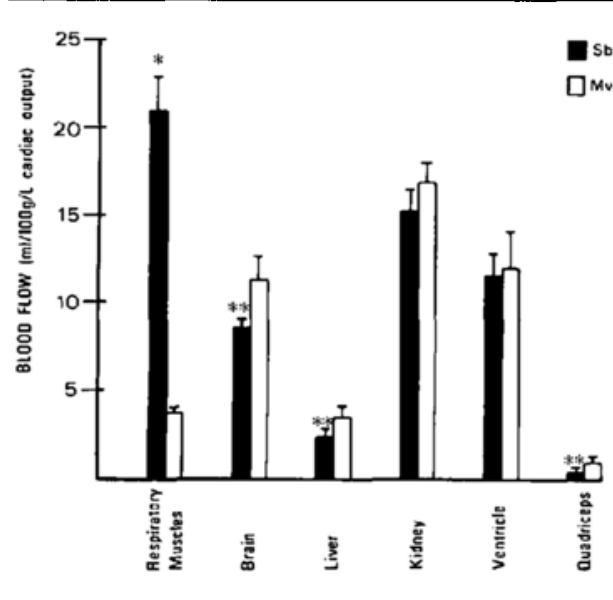

FIGURE 2 Comparisan of the fractional distribution of cardiac output during tamponade in the two groups of dogs. Bars represent SE. While the respiratory muscles received a significantly $\left({ }^{*}\right)$ greater portion of the cardiac output in the $\mathrm{Sb}$ group ( $p<0.001$ ), the brain, liver, and quadriceps muscles received significantly less $\left(^{* *}\right)(p<0.001) . S_{b}$ (black) $M_{v}$ (white).

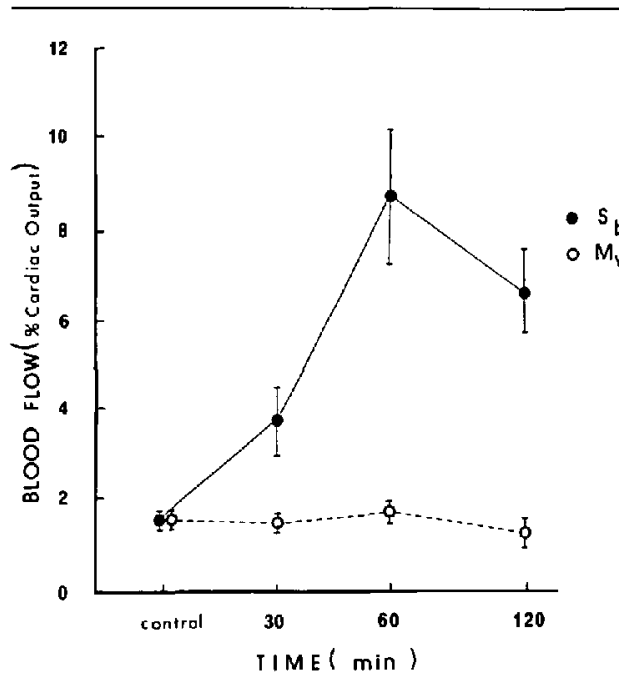

FIGURE 3 Total respiratory muscle blood flow in spontaneously breating $\left(\mathrm{S}_{\mathrm{b}}\right.$ ) dogs (solid circles) and mechanically ventilated $\left(\mathbf{M}_{v}\right)$ dogs (open circles). At all times during shock, the fractional distribution of cardiac output to respiratory muscles was higher in the $S_{b}$ group $(p<0.05)$ compared with the $M_{v}$ group. not only by other parts of the body, but also by the respiratory muscles. That is, despite the fact that these muscles received a large amount of blood, it was not adequate to meet their demands. Clearly they went into anerobic metabolism producing lactic acid and respiratory muscle fatigue which led to ventilatory failure and death of the animals. The conclusion is inescapable: the management of shock with hyperventilation is to put the respiratory muscles at rest to avoid both their failure and lactate production.

\section{Fatigue}

In general, respiratory muscle fatigue occurs whenever energy demand exceeds energy supply, as during high resistive breathing and/or hypoxia and low cardiac output states, both in cardiogenic or septic shock..$^{8,9,10}$

It should be noted that fatigue is characterized by a reduction in the force generating capacity of the muscles followed by a protracted period of exercise. ${ }^{8}$ Although the site of fatigue of skeletal muscles in general remains a subject of controversy, there is little doubt that in the respiratory muscles force failure will occur whenever the respiratory muscles do not receive adequate energy supply, as in the state of shock..$^{9.10}$ A classic example of such a failure is depicted in Figure 5 where both the phrenic discharge and the electrical activity of the diaphragm have increased in an animal model submitted to shock. At $140 \mathrm{~min}$, while the transdiaphragmatic pressure was substan-

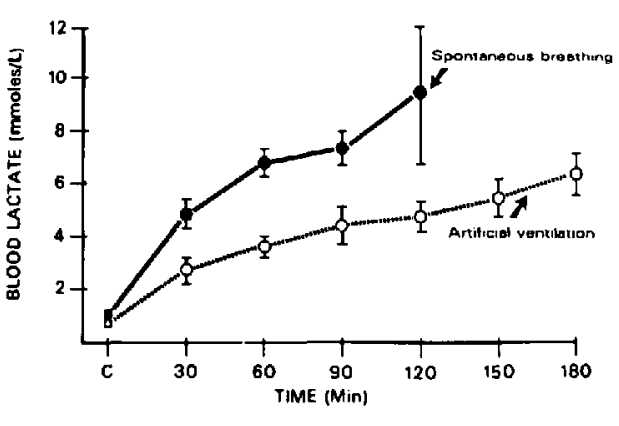

FIGURE 4 Time course of mean arterial blood lactate during cardiogenic shock. The first point represents control values. Bars are SE. 


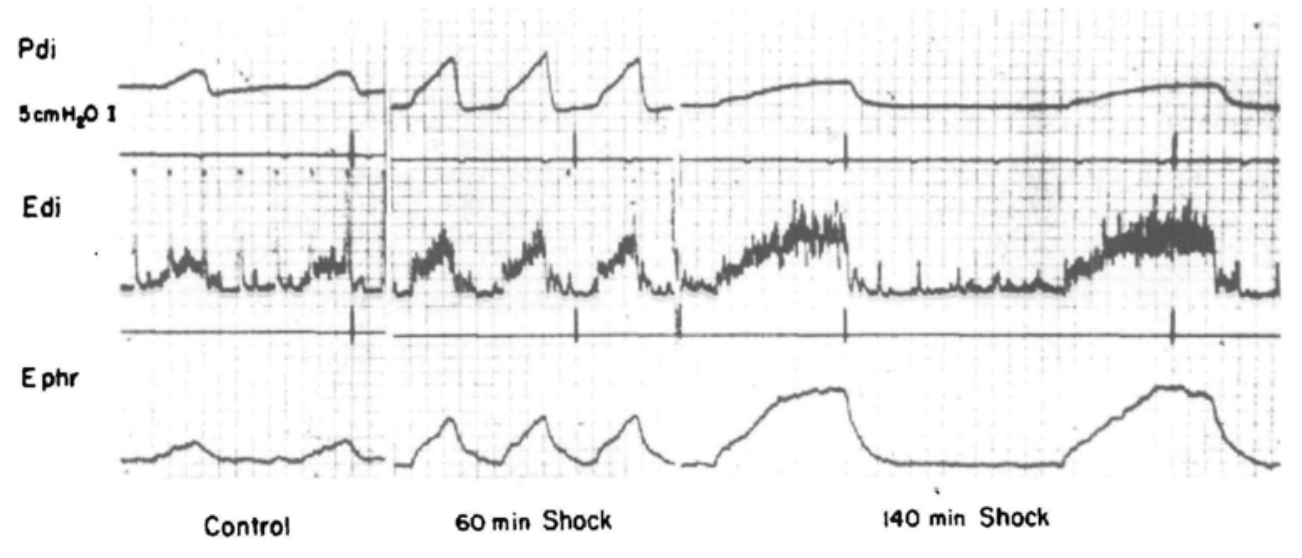

FIGURE 5 Evolution of transdiaphragmatic pressure (Pdi), integrated electrical activity of the diaphragm (Edi), and integrated electric activity of the phrenic nerve (Ephr) of a dog in cardiogenic shock. Left panel represents a control study; middle panel, a reading made 60 minutes after onset of cardiogenic shock; right panel, a reading made 140 minutes after onset of cardiogenic shock and just before death from respiratory arrest. While Edi and Ephr continue to increase, Pdi decreases (fattigue). The decrease in size of the ECG artifact on the Edi trace is a consequence of injection of saline into the pericardium.

tially reduced the excitation of the muscle was largely increased. It should be noted that all the animals submitted to either cardiogenic or septic shock died from respiratory failure while the circulation remained unchanged at the low level of cardiac output and decreased blood pressure. Thus, the sequence of events was an increase in blood flow to the diaphragm and the respiratory muscles, deprivation of the rest of the body of blood flow, a progressive increase of lactic acid, respiratory muscle fatigue, hypoventilation, hypoxaemia, hypercapnia, and finally, cardiac arrest. In contrast to this, early institution of mechanical ventilation prevents the shifting of blood flow from the vital organs, reduces lactic acidosis and avoids respiratory failure and death of the animal.

\section{Ventilatory failure}

Respiratory muscle fatigue is one of three pathways through which hypercapnic respiratory failure develops, fatigue will lead to alveolar hypoventilation and to $\mathrm{CO}_{2}$ retention. $1, \mathrm{tI}$

Alveolar hypoventilation with constant $\mathrm{CO}_{2}$ production and dead space volume depends on the total minute ventilation and the ratio of dead space to tidal volume (VD/VT), or both. During fatigue, as the muscle fails to develop adequate pressure, tidal volume is reduced and therefore both total minute ventilation decreases and VD/VT increases. We have found that in the acute state of hypercapnic respiratory failure - during cardiogenic and septic shock in dogs - the mechanisms responsible for $\mathrm{CO}_{2}$ retention are decreasing minute ventilation and increasing $V_{D} / V_{T}$ due to a decrease in $V_{T}$. The decrease in tidal volume is the result of a reduction in the inspiratory flow due to inability of the inspiratory muscles to develop adequate driving pressures. ${ }^{9-11}$

\section{Conclusion}

Placing the respiratory muscles at rest in patients in shock has a strong theoretical and experimental basis, both to avoid the complication of respiratory failure and to improve the prognosis. From our studies we may conclude that early institution of mechanical ventilation in shock will reduce respiratory muscle activity, will reduce respiratory muscle oxygen requirements, will avoid muscle fatigue and respiratory failure, will increase blood flow and oxygen availability to essential organs, will decrease the severity of lactic acidosis which may be associated with an improved prognosis and will decrease the demand of the failing myocardium whose own blood and oxygen supply may be critically reduced. 


\section{References}

1 Roussos C, Macklem PT. The respiratory muscles. N Engl J Med 1982; 307: 786-97.

2 Roussos C. Energetics. In: "The Thorax." Editors: C. Roussos, PT Macklem, C. Lenfant. Marcel Dekker 1985; 437-92.

3 Buchler B, Magder S, Roussos C. Effects of contraction frequency and duty cycle on diaphragmatic blood flow. J Appl Physiol 1985; 58: 265-73.

4 Robertson CH Jr, Foster GH, Johnson RL Jr. The relationship of respiratory failure to the oxygen consumption of, lactate production by, and distribution of blood flow among respiratory muscles during increasing inspiratory resistance. J Clin Invest 1977; 59: $31-42$.

5 Viires $N$, Sillye $G$, Aubier $M$, Rassidakis A, Roussos $C$. Regional blood flow distribution in dog during induced hypotension and low cardiac output. Spontaneous breathing versus artificial ventilation. J Clin Invest 1983; 72: 935-47.

6 Hussain S, Roussos C. Distribution of respiratory muscle and organ blood flow during endotoxin shock in dogs. J Appl Physiol 1985; 59: 1802-8.

7 Aubier $M$, Vitres $N$, Sillye $G$, Mozes $R$, Roussos $C$. Respiratory muscle contribution to lactic acidosis in low cardiac output state. Am Rev Respir Dis 1982; 126: 648-52.

8 Roussos C, Moxham J. Respiratory muscle fatigue. In: "The Thorax." Editors: C Roussos, PT Macklem, C Lenfant. Marcel Dekker 1985; 829-70.

9 Aubier $M$, Trippenbach $T$, Roussos $C$. Respiratory muscle fatigue during cardiogenic shock. J Appl Physiol 1981; 51: 499-508.

10 Hussain $S$, Simkus $G$, Roussos C. Ventilatory muscle fatigue, the cause of respiratory failure in septic shock. J Appl Physiol 1985; 58: 2033-40.

11 Roussos $C$. The failing ventilatory pump. Lung 1982; 160: 59-84. 